\title{
Hydrocarbon removal from bilgewater by a combination of air-stripping and photocatalysis
}

\author{
D. Cazoir*, L. Fine, C. Ferronato, J.-M. Chovelon \\ University Lyon 1, Lyon, F-69626, France, CNRS, UMR5256, IRCELYON, Institut de Recherches sur la Catalyse et l'Environnement de Lyon, Villeurbanne, F-69626, France
}

In order to prevent hydrocarbon discharge at sea from the bilge of ships, the International Maritime Organization (IMO) enacted the MARPOL $73 / 78$ convention in which effluents are now limited to those with maximum oil content of $15 \mathrm{ppmv}$. Thus, photocatalysis and air-stripping were combined for the hydrocarbon removal from a real oily bilgewater sample and an original monitoring of both aqueous and gaseous phases was performed by GC/ MS to better understand the process. Our results show that the hydrocarbon oil index [HC] can be reduced to its maximum permissible value of 15 ppmv (MARPOL) in only $8.5 \mathrm{~h}$ when photocatalysis and air-stripping are used together in a synergistic way, as against $17 \mathrm{~h}$ when photocatalysis is used alone. However, this air-assisted photocatalytic process emits a large quantity of volatile organic compounds (VOC) and, within the first four hours, ca. 10\% of the hydrocarbon removal in the aqueous phase is actually just transferred into the gaseous one. Finally, we highlight that the n-alkanes with a number of carbon atoms higher than $15\left(\mathrm{~N}_{\mathrm{C}}>15\right)$ are those which most decrease the rate of $[\mathrm{HC}]$ removal.

\section{Introduction}

Bilgewater, a polluted effluent with a smell of hydrocarbons and rotten eggs is a corrosive mixture from the bilge of ships that mainly originates from seawater infiltration and leakage of engine oil and fuel. Each year, two thousand tons of hydrocarbons from bilge are discharged at sea and this huge quantity is a major

Abbreviations: \%w, percentage (weight ratio); IMO, International Maritime Organization; ASP, air-stripping photocatalytic; MS, mass spectrometry; BTEX, benzene-toluene-ethylbenzene-xylene(s); MTBE, methyl tert-butyl ether; DAPR, diffused aeration photocatalytic reactor; NC, number of carbon atoms; ETBE, ethyl tert-butyl ether; $\mathrm{PAB}$, polyalkyl-benzene; $\mathrm{GC}$, gas chromatography; ppmv, part per million (volumic ratio); $H_{\mathrm{CC}}$, The Henry constant; RT, retention time; $\mathrm{HC} /[\mathrm{HC}]$, hydrocarbon/hydrocarbon oil index; UV, ultraviolet; IC, ionic chromatography; VOC/[VOC], volatile organic compound/concentration.

* Corresponding author. Tel.: +33472448405.

E-mail addresses: david.cazoir@ircelyon.univ-lyon1.fr, david.cazoir@hotmail.fr (D. Cazoir). pollution problem for marine ecosystems and more or less directly for human health, causing the International Maritime Organization (IMO) to enact MARPOL 73/78 convention in which effluents from ships are now limited to a maximum oil content of $15 \mathrm{ppmv}$ [1].

However, because on-board storage of bilgewater is not always feasible, remediation is required and different kinds of treatment have been proposed. Of these, physical processes are currently the most commonly-used techniques. The oil-in-water separators with which ships have to be equipped [2] make the remediation of a huge quantity of oil possible. However, there are still some engineering problems for this kind of reactor and, in the presence of some particles or coupled with a failed detector, discharge at sea may take place even when the oil content is higher than 15 ppmv. Other problems are (i) the extremely long time required for the separation process which can favour the irreversible dissolution of organic materials, and (ii) the presence of detergents that promote emulsion formation. Filtration techniques, sometimes combined with reverse osmosis [3], have been proposed to enhance the separation 


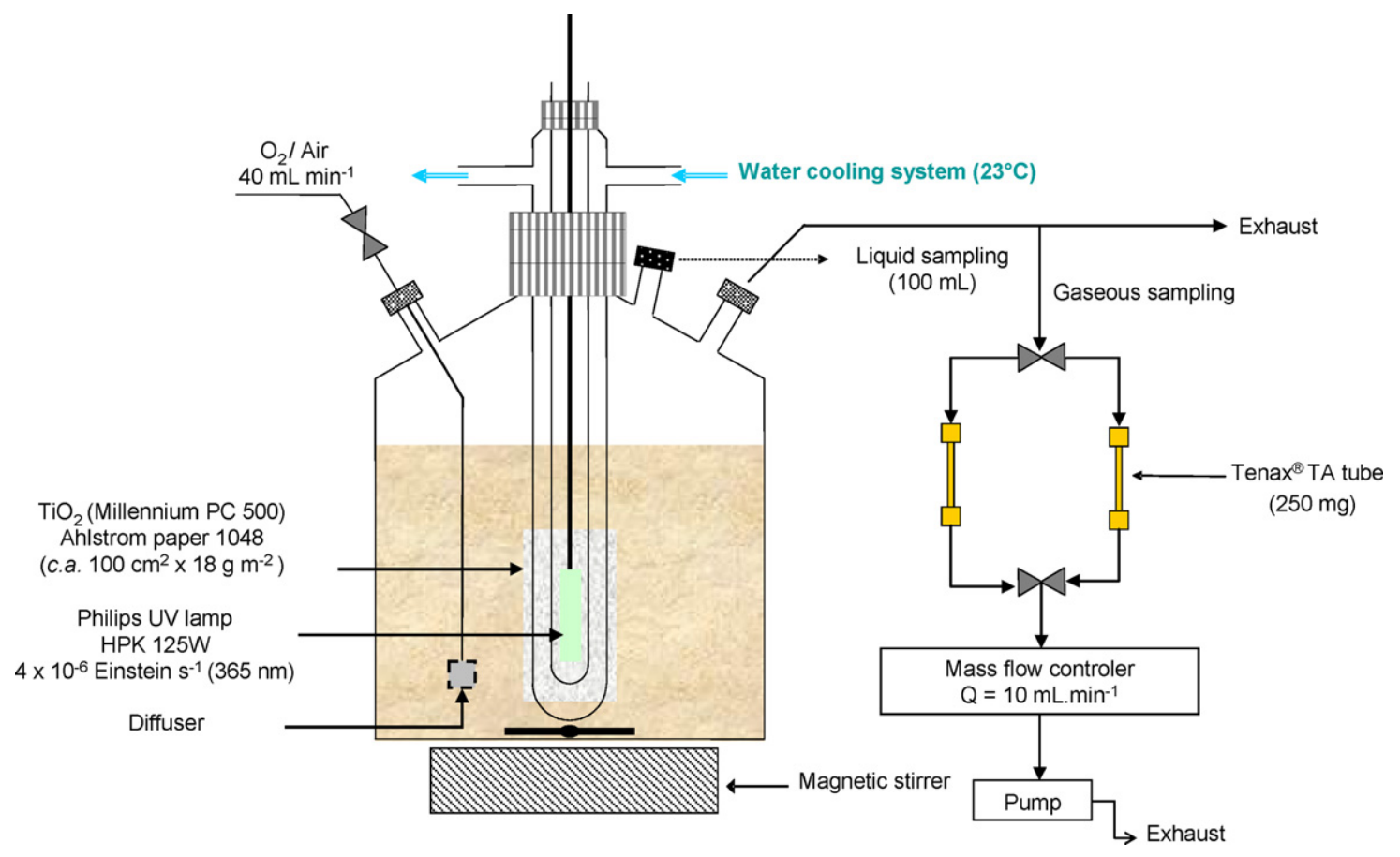

Fig. 1. Schematic of the $1 \mathrm{~L}$ batch photoreactor.

of aqueous and organic phases [4,5]. Such physical processes do not degrade the pollutants but only separate them.

To counter this, biological processes have been proposed as a degrading method for the removal of hydrocarbons from this kind of effluent. A pilot bioreactor containing microorganisms was tested aboard the Caps Lobos MARAD motor vessel in combination with a physical process. Results showed that more than $300 \mathrm{~m}^{3}$ could be treated each month in this way [6]. More recently, the presence of some Pseudomonas species in bilge oil was also highlighted and biodegradation was studied using these organisms [7]. These bacteria are able to degrade $70 \%$ of the hexane-extractable organics and $90 \%$ of the aromatic compounds. The efficiency of this process can also be improved by using cyclodextrins as carrier molecules for increasing the water solubility of the low water soluble hydrocarbons [8]: e.g. after $120 \mathrm{~h}$ of treatment, only $15 \%$ of hexadecane remained undegraded as against $43 \%$ when cyclodextrins were not used.

Many other techniques have been studied in order to degrade hydrocarbons from bilgewater. We can cite for example, wet air oxidation [9], electrochemical purification $[10,11]$ and photocatalysis $[12,13]$.

Photocatalysis is an advanced oxydation processes with which any sample contaminated by an organic substrate can be remediated until mineralisation, by using a semiconductor $\left(\right.$ e.g. $\left.\mathrm{TiO}_{2}\right)$ and a source of photons like a UV lamp or sunlight [14-17]. Promising results have been obtained for polar compounds in water [18-20] as well as for hydrocarbons $[13,18,21-23]$. In order to treat bilgewater samples, Karakulski et al. [12] coupled a filtration process to a photocatalytic one and achieved a total decomposition of the oil after $50 \mathrm{~h}$ of ultrafiltration (the chemical oxygen demand was removed by ca. $96 \%$ in the permeate) followed by 2 h of UV illumination using a $\mathrm{K}-\mathrm{TiO}_{2}$ photocatalyst. Lair also worked on the treatment of bilgewater by photocatalysis but limitations due to a lack of dissolved oxygen appeared since $\left[\mathrm{O}_{2}\right]$ did not exceed $1 \mathrm{mg} \mathrm{L}^{-1}$ [13]. Indeed, the photocatalytic process needs a constant supply of oxygen toward the photocatalyst in order to oxidize all the organic compounds and to avoid undesirable electron-hole recombination [24].
Aeration therefore appears to be a good means of improving photocatalytic performance in this case. In addition, aeration favours the air-stripping phenomenon which is based on the principle that dissolved volatile compounds can be carried away by a gas stream that shifts the equilibrium from aqueous to gaseous phase. The gas-liquid partitioning behaviour of volatile solutes obeys Henry's law, which states that the partial pressure of a component in the gas phase is proportional to its concentration in the liquid phase under equilibrium conditions. The dimensionless form of Henry's law constant $\left(H_{\mathrm{CC}}\right)$ can be expressed as:

$H_{\mathrm{CC}}=\frac{C_{i, G}}{C_{i, L}}$

where $C_{i, L}$ and $C_{i, G}$ are the concentrations of the compound in moles per volume of solution and gas, respectively. Staudinger and Roberts published a compilation of various $H_{\mathrm{CC}}$ of organic compounds [25] which has proved to be very useful when studying the air-stripping phenomenon, the latter being already used in water treatment [26] especially in the case of surfactant-containing wastewater $[27,28]$.

This work reports on the combination of photocatalysis and airstripping for bilgewater remediation. Since aeration would play various roles during the photocatalytic treatment, a synergistic effect is expected, and in order to better evaluate the efficiency of the combined process, the behaviour of both aqueous and gaseous phases were monitored with an original experimental set up.

\section{Experimental}

\subsection{Bilgewater preparation}

Once received from a French military ship, samples were first well mixed during one hour and then settled for $30 \mathrm{~min}$ in order to obtain after separation (i) a realistic and reproducible oily aqueous phase of bilgewater (hereinafter called the bilgewater sample) from one hand and (ii) a 100\% organic phase from another hand. So the bilgewater sample was used immediately to prevent instability during any storage. Where needed, different initial concentrations 
were obtained by mixing the bilgewater sample with the $100 \%$ organic phase and adjusting the ratio between both phases and the times of contact during mixing and of settle for the final separation.

\subsection{The diffused aeration photocatalytic reactor (DAPR)}

Experiments were conducted at room temperature in a cylindrical $1 \mathrm{~L}$ batch liquid phase reactor $(\varnothing 120 \mathrm{~mm})$ in which the photocatalytic reaction and the air-stripping take place simultaneously (Fig. 1).

Near-UV radiation was emitted by a lamp inserted in the centre of the Pyrex annular vessel. The lamp was enclosed in a double jacket in order to provide temperature control via water circulation. The light source was a Philips HPK $125 \mathrm{~W}$ mercury lamp with a maximum of intensity around $365 \mathrm{~nm}$ : a photon flux of ca. $4 \times 10^{-6}$ Einstein $\mathrm{s}^{-1}$ was measured with a Vilbert Lourmat W89325 radiometer (coupled with a W89328 sensor at $365 \mathrm{~nm}$ ). The photocatalytic medium used was a $15 \times 6.5 \mathrm{~cm}^{2}$ commercial nonwoven fibrous paper 1048 (Ahlstrom, France) coated with $\mathrm{TiO}_{2}$ PC500 (100\% anatase, BET area: $320 \mathrm{~m}^{2} \mathrm{~g}^{-1}$, crystal size: $5-10 \mathrm{~nm}$, $18 \mathrm{~g}_{\mathrm{TiO}_{2}} \mathrm{~m}^{-2}$ ). This was directly fixed on the lamp jacket and was UV-pretreated in deionized water for an entire night before being used in any photocatalytic degradation.

Meanwhile, the air-stripping process was ensured by a diffuser from which a compressed air flow was bubbled into the solution. The gas flow rate was adjusted to $40 \mathrm{~mL} \mathrm{~min}^{-1}$ using a mass flow controller (Brooks, 5850 S series) and the reactor headspace was partially opened to avoid overpressure, and compounds emitted in the gaseous phase from the bubbled sample were then purged outside, passing through stainless steel lines before being analysed.

Finally, the concentration of dissolved oxygen $\left[\mathrm{O}_{2}\right]$ in the aqueous phase could be measured when needed with a WTW Oxi 315i equipment coupled with a WTW DurOx 325 sensor.

\subsection{Sampling and analysis of the aqueous phase}

\subsubsection{GC analysis: determination of the hydrocarbon oil index [HC]}

Gas chromatography/mass spectrometry (GC/MS) analyses were performed to identify and quantify the different organic pollutants. GC/MS determinations were carried out on a PerkinElmer Clarus 500 system using a capillary Elite 5MS column and the analysis conditions are reported in Table 1 . The mass spectrometer was operated in total ion current mode and identification of compounds was made by comparison of a high probability match with mass spectra reported in the library of the National Institute of Standards and Technology (NIST).

Samples for analysis were prepared according to the main steps described in ISO 9377-2:2000, viz. (i) $9 \mathrm{~g}$ of a salt $\left(\mathrm{MgSO}_{4} \cdot 7 \mathrm{H}_{2} \mathrm{O}\right)$ were added to the volume of the sample to be analysed $(100 \mathrm{~mL})$; (ii) $\mathrm{pH}$ was adjusted with a few droplets of $\mathrm{HCl}$ (q.s. $\mathrm{pH} 2$ ); (iii) the sample was extracted with a total volume of $50 \mathrm{~mL}$ of hexane; (iv) a solvent evaporation under vacuum was performed in order to concentrate the organic compounds in the final sample in which (v) an internal standard of 1-bromododecane BD (Fluka, 97\%) was added before GC analyses (ca. $40 \mathrm{mg}$ of a 1000 ppmv solution in hexane).

According to the International Maritime Organization that refers to the ISO 9377-2:2000 standard [29,30], the hydrocarbon oil index $[\mathrm{HC}]$ was determined by the summation of all the concentrations of the different compounds eluted between n-decane and n-tetracontane (expressed in ppmv undecane equivalent):

$$
[\mathrm{HC}]=\sum_{i=\mathrm{C}_{10} \mathrm{H}_{22}}^{\mathrm{C}_{40} \mathrm{H}_{82}} C_{i}
$$

Experimental errors are mainly due to the organics extraction-concentration yield that depends on compound properties and has been valued at $20 \%$ max. (chosen from the extraction yield measured for the most volatile one, i.e. the decane).

\subsubsection{Determination of the inorganic composition}

Inorganic composition was determined by ionic chromatography (IC) in which anions were analysed using a Dionex DX-120 ion-chromatograph equipped with a Dionex AS 40 autosampler, a conductivity detector and an IonPac AS14A $(4 \times 250 \mathrm{~mm})$ anion exchange column. The eluent was an aqueous solution of $\mathrm{Na}_{2} \mathrm{CO}_{3} / \mathrm{NaHCO}_{3}(8 / 1 \mathrm{mM})$. Analysis of cations was performed using the same apparatus except for the column (CS12A, $4 \times 250 \mathrm{~mm}$ ) and eluent $\left(\mathrm{H}_{2} \mathrm{SO}_{4}, 20 \mathrm{mM}\right)$. All elutions have been carried out at a flow rate of $1 \mathrm{~mL} \mathrm{~min}^{-1}$.

As for bicarbonate concentration, this was determined by an acid-base dosage using a hydrochloric acid solution (0.1 M).

\subsection{Sampling and analysis of the gaseous phase: determination of [VOC]}

Compounds emitted in the gaseous phase were collected and concentrated for $10 \mathrm{~min}$ at a flow rate of $10 \mathrm{~mL} \mathrm{~min}^{-1}$ on solid sorbent tubes packed with $250 \mathrm{mg}$ of Tenax ${ }^{\circledR}$ TA (Supelco). Desorption of the adsorbed compounds was carried out on an Automated Thermal Desorption unit (Turbomatrix, PerkinElmer) coupled to the same gas chromatography/mass spectrometry (GC/MS) equipment as that used for the aqueous phase analysis and the analytical conditions are reported in Table 1.

All the concentrations are given in ppmv undecane equivalent. A VOC index [VOC] was then determined by integrating all the concentrations of the different compounds eluted between the 2-methoxy-2-methyl-propane (MTBE) and the ultimate one adsorbed on the Tenax ${ }^{\circledR}$ TA and analysed by GC. Experimental errors, since they are mainly due to flow rate fluctuations during sampling, have been valued at $10 \%$.

\section{Results and discussion}

\subsection{Characterisation of the aqueous phase}

Fig. 2 presents the GC chromatogram of the aqueous phase of a bilgewater sample whose hydrocarbon oil index [HC] is $c a$. 44 ppmv. A typical cut of gas oil (i.e. diesel) centred on pentadecane can be observed in which linear alkanes represent $35 \mathrm{wt} \%$ of the total $[\mathrm{HC}]$. Among this n-alkane family, the last compound analysed is tricosane $\left(\mathrm{C}_{23} \mathrm{H}_{48}\right)$; heavier n-alkanes, since they are less soluble in water, are expected to remain in the organic phase which was separated beforehand from the collected sample (see Section 2.1). Branched-alkanes form the second largest family of compounds, even if they are not always well identified. Aromatic molecules such as naphthalene derivatives and alkyl- and polyalkyl-benzenes (PABs) have also been detected. They represent almost $10 \mathrm{wt} \%$ of the $[\mathrm{HC}]$ without taking into account the BTEXs family (benzenetoluene- ethylbenzene- $\mathrm{o}, \mathrm{m}$ and $\mathrm{p}$-xylene) that are not concerned by the MARPOL regulations as they are eluted before decane. Polar compounds such as sulphur-containing and oxygenated hydrocarbons are also present but the calculation of their real contribution to the $[\mathrm{HC}]$ cannot be safely done because of a lower extraction yield.

As can be seen in Table 2, the inorganic ion composition of bilgewater is similar to that of seawater [31] with a large quantity of sodium and chloride ions $\left(10 \mathrm{~g} \mathrm{~L}^{-1}\right.$ and $20 \mathrm{~g} \mathrm{~L}^{-1}$ respectively). Likewise, we notice the presence of $\mathrm{K}^{+}, \mathrm{Mg}^{2+}, \mathrm{Ca}^{2+}$ ions with concentrations of same order of magnitude as measured in seawater. 
Table 1

Analytical conditions for the GC-MS analyses of the aqueous and gaseous phases.

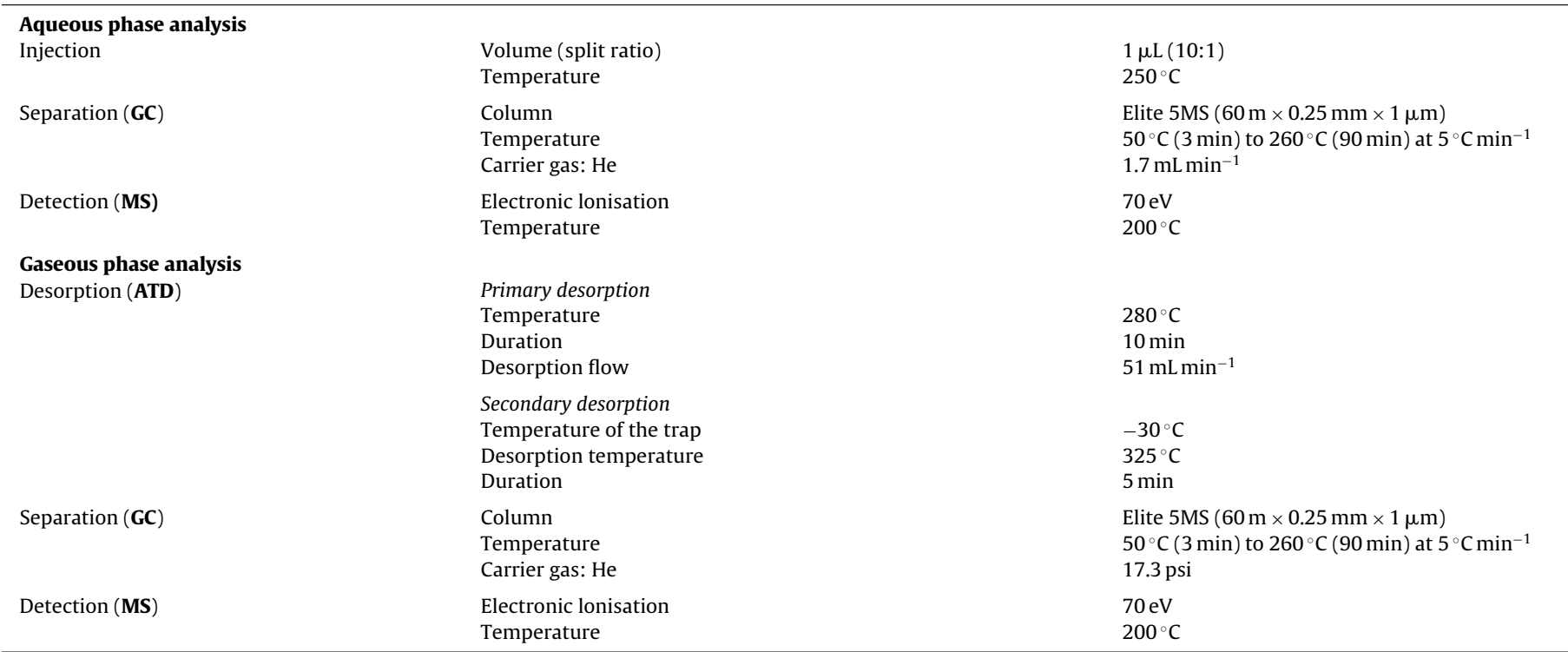

Table 2

Inorganic composition and physico-chemical properties of the bilgewater sample comparison with seawater values [31] into parentheses.

\begin{tabular}{|c|c|c|c|}
\hline \multicolumn{4}{|c|}{ Inorganic composition $\left(\mathrm{g} \mathrm{L}^{-1}\right)$} \\
\hline $\mathrm{Na}^{+}$ & $10(10)$ & $\mathrm{Cl}^{-}$ & $20(20)$ \\
\hline $\mathrm{K}^{+}$ & $0.3(0.4)$ & $\mathrm{SO}_{4}{ }^{2-}$ & $0.4(3)$ \\
\hline $\mathrm{Ca}^{2+}$ & $0.2(0.4)$ & $\mathrm{NO}_{3}^{-}$ & $<\mathrm{SD}(0.02)$ \\
\hline $\mathrm{Mg}^{2+}$ & $0.9(1)$ & $\begin{array}{l}\mathrm{NO}_{2}^{-} \\
\mathrm{HCO}_{3}^{-}\end{array}$ & $\begin{array}{l}<\mathrm{SD} \\
2(0.1)\end{array}$ \\
\hline \multicolumn{4}{|c|}{ Physico-chemical properties (at $25^{\circ} \mathrm{C}$ ) } \\
\hline $\mathrm{PH}$ & $7.8(7.5-8.4)$ & {$\left[\mathrm{O}_{2}\right]\left(\mathrm{mg} \mathrm{L}^{-1}\right)$} & 0.8 \\
\hline
\end{tabular}

However, differences can be observed for sulphate and nitrate concentrations that are lower than expected. This can easily be explained as we measured a dissolved oxygen concentration $\left[\mathrm{O}_{2}\right]$ of about $0.8 \mathrm{mg} \mathrm{L}^{-1}$ : bilgewater samples are reducing media and the elements sulphur and nitrogen are probably more present in their reduced forms. Finally, our $\mathrm{pH}$ measurements gave a value of 7.8 , i.e. just like that of a seawater sample in the same conditions of pressure and temperature.

\subsection{Characterisation of the gaseous phase}

The composition of the gaseous phase, while aeration is turned ON, is illustrated in Fig. 3. [VOC] is about 110 ppmv and alkanes (linear and branched) represent $c a .45 \%$ of this concentration. The heaviest n-alkanes $\left(\mathrm{N}_{\mathrm{C}}>13\right)$ are not purged into the gaseous phase but this result is not surprising as the phenomenon that occurs here is mainly governed by Henry's law. BTEXs, and more generally PABs, are also detected. Benzene which was not clearly identified in the aqueous phase is now well characterized and its behaviour can be followed. In the same way, detection of ethers such as MTBE

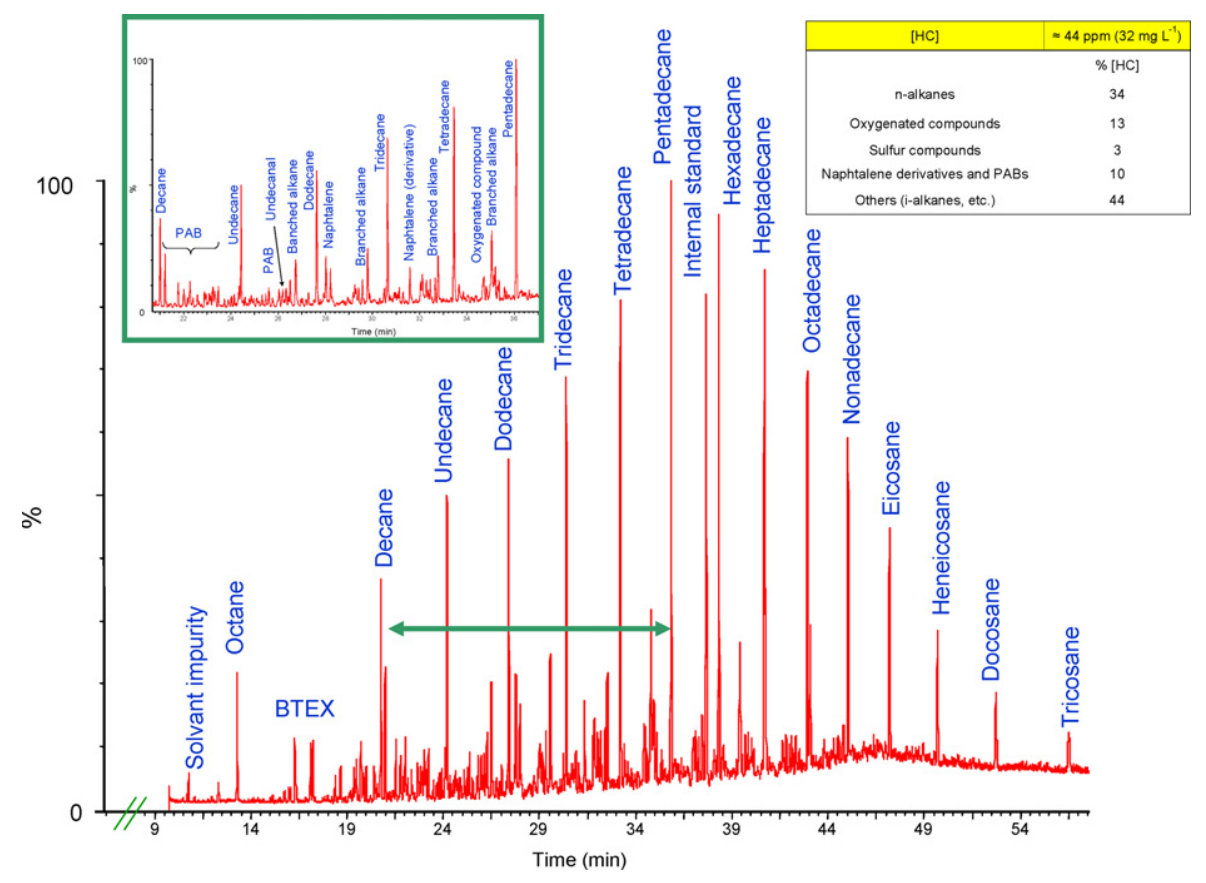

Fig. 2. Aqueous phase analysis of the bilge water sample (GC-MS). 


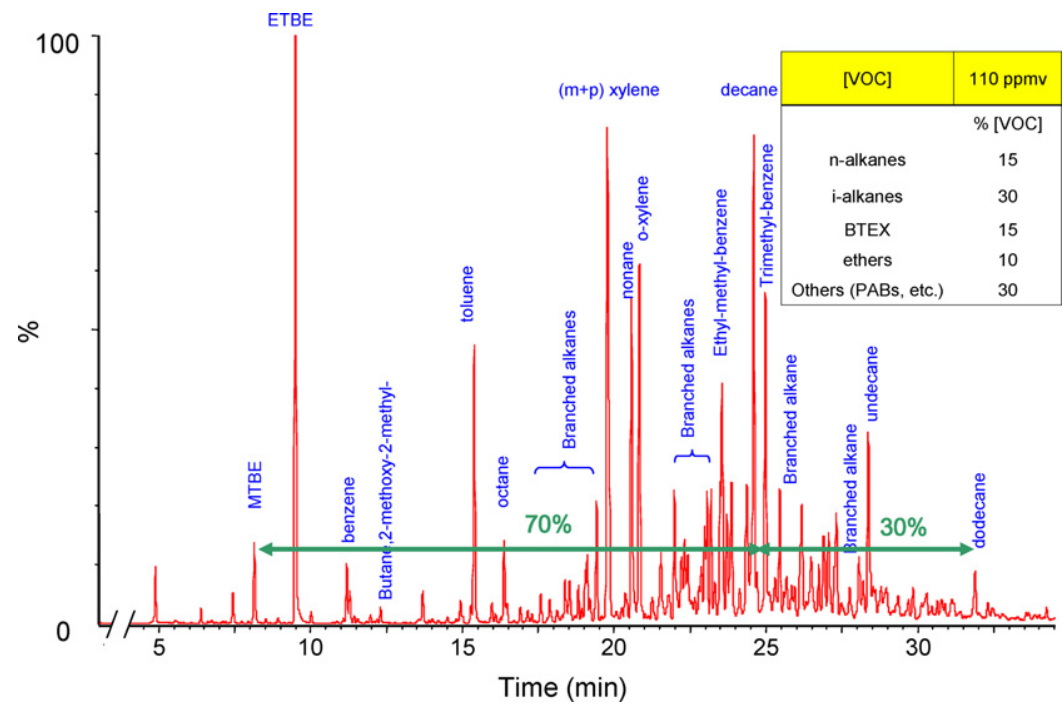

Fig. 3. Gaseous phase analysis of the bilge water sample. during the first $10 \mathrm{~min}$ of air supply (GC-MS)

and ETBE (respectively methyl- and ethyl- tert-butyl ether) is very interesting; due to their high solubility in water, they were not extracted with hexane and therefore not detected in the aqueous phase. On the contrary, Fig. 3 indicates that they represent more than $10 \%$ of the VOC transferred from the aqueous phase into the gaseous one. Moreover, these compounds are known to be a real problem for groundwater where they accumulate, so the study of their behaviour is relevant. Finally, it must be pointed out that $70 \%$ of the VOC amount comes from pollutants that are eluted before decane. In other words, only $30 \%$ comes from pollutants that have to be related to the hydrocarbon oil index $[\mathrm{HC}]$ of the aqueous phase.

\subsection{Air-stripping-photocatalytic (ASP) treatment}

\subsection{1. $[\mathrm{HC}]$ removal in the aqueous phase and synergistic effect}

Since bilgewater samples had low dissolved oxygen content, air was supplied during the process and we studied the effect of this air-striping photocatalytic (ASP) treatment on the hydrocarbon removal. In Fig. 4, the initial rate was reported as a function of the initial concentration during both photocatalytic and ASP treatments. Fig. 4 clearly indicates an enhancement of the rate of the $[\mathrm{HC}]$ removal when air is supplied. The kinetic constant of apparent first order is twice as large in the ASP experiments as in the photocatalytic ones $\left(20 \times 10^{-4} \mathrm{~min}^{-1}\right.$ vs. $\left.9 \times 10^{-4} \mathrm{~min}^{-1}\right)$ so aeration seems to improve the removal efficiency. Oxygen plays two important roles in photocatalysis: (i) it prevents the electron-hole recombination (reaction (R2)) by scavenging the photogenerated electron (reaction (R3)); (ii) during reaction $\mathrm{R} 3$, its reduction produces

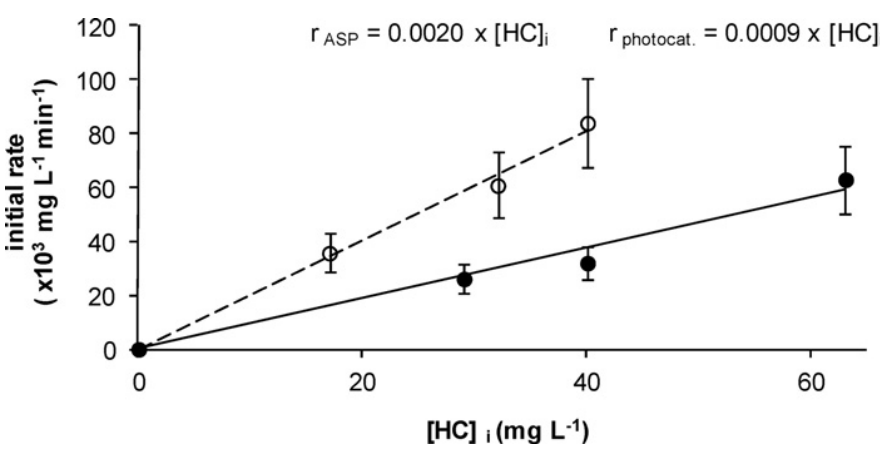

Fig. 4. Influence of treatment on the initial rate of $[\mathrm{HC}]$ removal. ( Photocatalysis $\bigcirc$ photocatalysis + air-stripping.) reactive oxygen species such as $\mathrm{O}_{2}{ }^{\bullet-}, \mathrm{HO}_{2} \bullet$ (and $\mathrm{H}_{2} \mathrm{O}_{2}$ which photodissociates to $2 \mathrm{OH}^{\bullet}$ ) which improve the oxidation efficiency. It is to be noted that without a sufficient amount of $\mathrm{O}_{2}$ and with a high UV intensity lamp, reaction (R3) is not fast enough to prevent the non desired recombination reaction (R2) because of rate limitations in oxygen diffusion and, consequently, reaction (R4) cannot occur sufficiently for producing the strong oxidant radical $\mathrm{OH}^{\bullet}$.

$\mathrm{TiO}_{2}+h v \rightarrow \mathrm{e}^{-}+\mathrm{h}^{+}$

$\mathrm{e}^{-}+\mathrm{h}^{+} \stackrel{k_{1}}{\longrightarrow}$ recombination(+heat)

$\mathrm{e}^{-}+\mathrm{O}_{2} \stackrel{k_{2}}{\longrightarrow} \mathrm{O}_{2}^{\cdot-} \stackrel{\mathrm{H}+}{\longrightarrow} \mathrm{HO}_{2}^{\cdot}$

$\mathrm{h}^{+}+\mathrm{OH}^{-} \stackrel{k_{3}}{\longrightarrow} \mathrm{OH}^{\bullet}$

In a previous study, authors report that when the aeration is not sufficient, $\left[\mathrm{O}_{2}\right]$ decreases during a photocatalytic treatment [24]. The increase of $\left[\mathrm{O}_{2}\right]$ observed in Fig. 5 during our ASP experiments indicates that here, a sufficient amount of oxygen is supplied to avoid diffusion limitations, so the oxidation efficiency is improved. At the same time, Fig. 5 follows the $[\mathrm{HC}]$ removal during the ASP treatment and we can thus determine the time required to reduce the initial hydrocarbon oil index to its 15 ppmv limited value (MAR$\mathrm{POL})$. In this figure, $[\mathrm{HC}]$ decreases with an exponential decay, i.e.

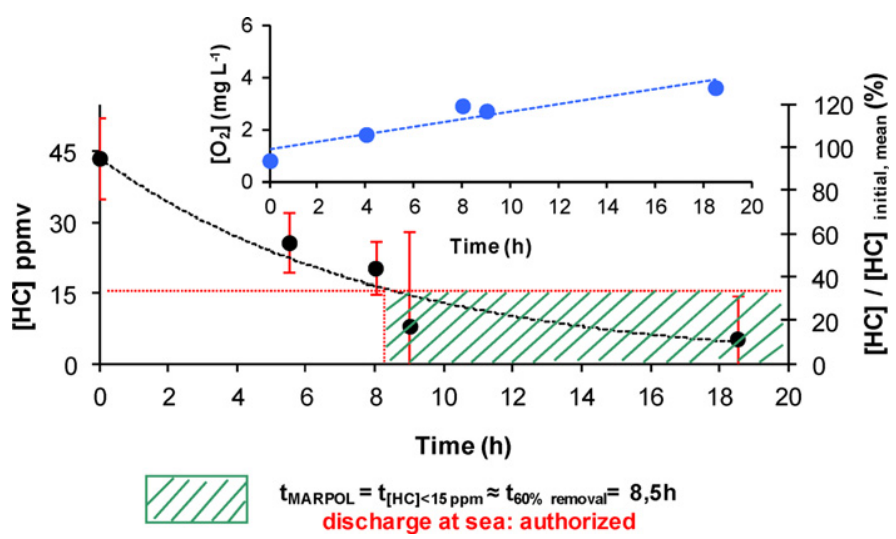

Fig. 5. HC removal and determination of $t_{\text {MARPOL }}$ during the ASP treatment: (blue points: monitoring of $\left[\mathrm{O}_{2}\right]$ during the process). (For interpretation of the references to color in this figure legend, the reader is referred to the web version of the article.) 


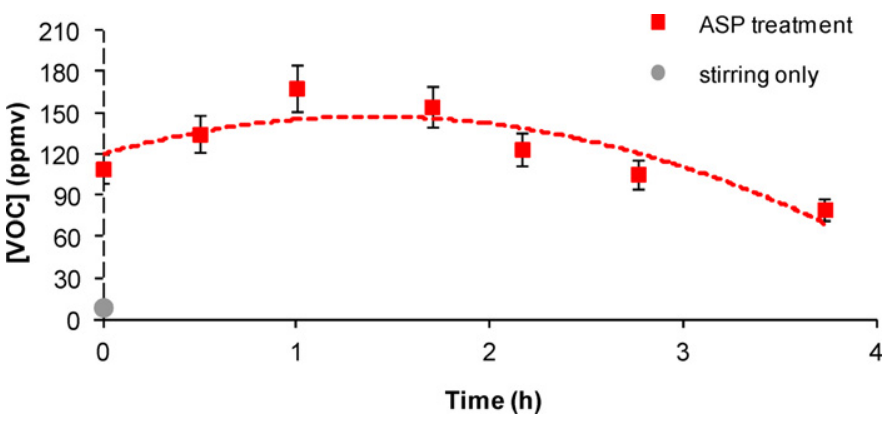

Fig. 6. $[\mathrm{VOC}]$ monitoring during the air-stripping photocatalytic (ASP) process.

the apparent rate constant is not dependent on the initial concentration (kinetic of pseudo first order according to the last results in Fig. 4). Thus, we can highlight that a removal of $\mathrm{ca} .60 \%[\mathrm{HC}]$ which is achieved in $8.5 \mathrm{~h}\left(t_{50 \%} \sim 6.5 \mathrm{~h}\right)$ is required to reduce the oil content from $44 \mathrm{ppmv}$ to $15 \mathrm{ppmv}$ (MARPOL). This time of $60 \%$ removal $\left(t_{60 \%}\right)$ thus defines the $t_{\text {MARPOL }}$ that we will use later for the identification of the most resistant compounds. In comparison, when photocatalysis is used alone, a simple calculation ${ }^{1}$ from the results shown in Fig. 4 indicates that in the best cases $t_{\text {MARPOL }}$ is $c a .17 \mathrm{~h}$. It is to be noted that error bars in Fig. 5 are calculated from the experimental error due to the extraction/evaporation step (max. $20 \%$ ). Since each point in Fig. 5 is the result of a specific experiment for which the initial concentration can be slightly different from the mean ( $44 \mathrm{ppmv}$ ), we added to these experimental errors, the deviation between initial concentration and mean concentration.

Finally, we measured the synergistic effect by using the following equation:

$S(\%)=\frac{k_{\mathrm{ASP}\left(\mathrm{TiO}_{2}+\mathrm{UV}+\text { aeration }\right)}}{k_{\text {photocatalysis }\left(\mathrm{TiO}_{2}+\mathrm{UV}\right)}+k_{\text {air-stripping }\left(\mathrm{TiO}_{2}+\text { aeration }\right)}}$

where $k$ is the pseudo first order rate constant under the experimental conditions indicated in subscripts. In each case (airstripping, photocatalytic and ASP experiments), a pseudo first order kinetic was observed so that the experimental constants $k$, which do not depend on the initial concentration, were reported in Eq. (3) giving a $S \%$ value equal to $105 \%$. As we point out, $S \%$ is not so different from the value of zero-synergy (100\%) and the better efficiency observed during the ASP treatment is only due to a slight synergistic effect.

\subsection{2. [VOC] monitoring in the gaseous phase}

Fig. 6 reports the evolution of the VOC concentration [VOC] during the treatment. Without aeration a very small concentration was measured at $t=0 \mathrm{~h}$ but this cannot be neglected as it is sufficient to pollute the ambient air (smell of hydrocarbons and other odorous compounds). By introducing air flow, [VOC] increases drastically within the first hour until a concentration of $c a .170 \mathrm{ppmv}$ (first part of the curve). Then, [VOC] decreases and reaches $c a .80$ ppmv after $4 \mathrm{~h}$ of reaction (second part of the curve). During the first part of the curve, the concentration increases mainly due to a stripping effect of the lightest molecules either initially present in the solution or generated during the treatment. In the second part, the decrease is due to the depletion of these molecules, since the amount of organic compounds in the solution decreases by the action of the combined treatment.

Fig. 7 shows the concentration evolution of different VOCs in the gaseous phase. Most of them (decane, undecane, dodecane and ethers) follow the global behaviour of [VOC], i.e. an initial increase

${ }^{1} t_{60 \%}=\frac{\ln (1 / 0.4)}{0.0009}$. and then a decrease. However, in the case of BTEX and octane, only a decrease of their concentration was observed. The behaviour of a compound essentially depends on three characteristics, viz. the solubility, the $H_{\mathrm{CC}}$ value and the photocatalytic reactivity. BTEX are easily removed by photocatalysis and their low $H_{\mathrm{CC}}$ values $(\sim 0.2$ for BTEX according to [25]) as well as their relatively good solubility in water suggest that their extraction by air-stripping from the aqueous phase is more difficult than their diffusion towards the photocatalytic paper and their oxidation. For these reasons, their concentrations only decrease. On the contrary, $H_{\mathrm{CC}}$ values for nalkanes are $\mathrm{ca}$. three orders of magnitude greater $\left(H_{\mathrm{CC}} \sim 100\right)$ and these compounds are not very reactive in photocatalysis. Thus, they are first extracted from the aqueous phase by air-stripping and their concentration increases slowly as a function of their diffusion properties. Then, the concentrations in the gaseous phase decrease because of the depletion of organic materials in the aqueous phase due to both air-stripping and photocatalysis. An exception can be formulated in the case of octane for which the concentration only decreases; in comparison with the other n-alkanes, its higher solubility reduces its air-stripping sensibility and the photocatalytic pathway is then favoured. By regarding the behaviour of MTBE and $\operatorname{ETBE}\left(H_{\mathrm{CC}} \sim 0.02\right)$, it is quite surprising to first observe an increase in their concentrations. Indeed, air-stripping is not expected to be as efficient as for compounds with $H_{\mathrm{CC}}$ greater than 0.05 [32] and thus, as for BTEX and for any other polar compounds, their concentration should only decrease. However, it has been reported that the photocatalytic removal efficiency of ethers like MTBE decreases in the presence of BTEX [33] and other strongly adsorbed compounds (e.g. n-alkanes - see next section). This may explain why these compounds are more affected by the air-stripping phenomenon and why their concentrations first increase (due to diffusion phenomena) and then decrease like that of n-alkanes do.

\subsubsection{Air-stripping discussion}

The integration of the curve in Fig. 6 indicates that the total mass of compounds lost by stripping follows a quasi linear tendency (Fig. 8). We can observe in Fig. 8 that after $4 \mathrm{~h}$ of ASP treatment, $5 \mathrm{mg}$ of VOC are purged into the gaseous phase: $70 \%$ of these VOC are eluted before decane whereas only $30 \%$, i.e. $1.5 \mathrm{mg}$, are eluted after this reference compound (Fig. 3). Thus, considering that during the same period [HC] decreases from 32 to $16 \mathrm{mg} \mathrm{L}^{-1}$ in the aqueous phase (16 mg treated in our 1 L DAPR), this last result indicates that ca. $10 \%$ of the heaviest compounds removed are just transferred into the gaseous phase.

It must be pointed out that the importance of air-stripping is clearly dependent on the Henry's constant of the molecule. In this constant, two properties are important, viz. solubility and volatility. Hence, the contribution of air-stripping in the HC removal from the aqueous phase depends on the molecule itself. For example, $60 \%$ of the initial mass of decane is lost by air-stripping after $4 \mathrm{~h}$ of treatment as against less than $40 \%$ for undecane (Table 3 ). Obviously, this contribution decreases when the volatility decreases. In the case of n-alkanes, when the length of the hydrocarbon chain $\left(\mathrm{N}_{\mathrm{C}}\right)$ increases, volatility and solubility both decrease so that the contribution of air-stripping also decreases. This contribution can even reach zero when $\mathrm{N}_{C}$ is higher than that of tridecane, the latter being only slightly purged into the gaseous phase. In Table 3, we also observe that, in comparison with the classic photocatalytic process, (1) the total removal by ASP treatment is enhanced in the cases of both decane and undecane; (2) the amount removed by photocatalysis is enhanced in the combined process (from $16 \%$ to $43 \%$ in the case of undecane). Finally, the photocatalytic removal of decane is not significantly enhanced by aeration. Indeed, decane removal is about $23-24 \%$ in both cases, i.e. during ASP treatment or during a classical photocatalytic process; when using ASP treatment, decane is drastically sent into the gaseous phase and 

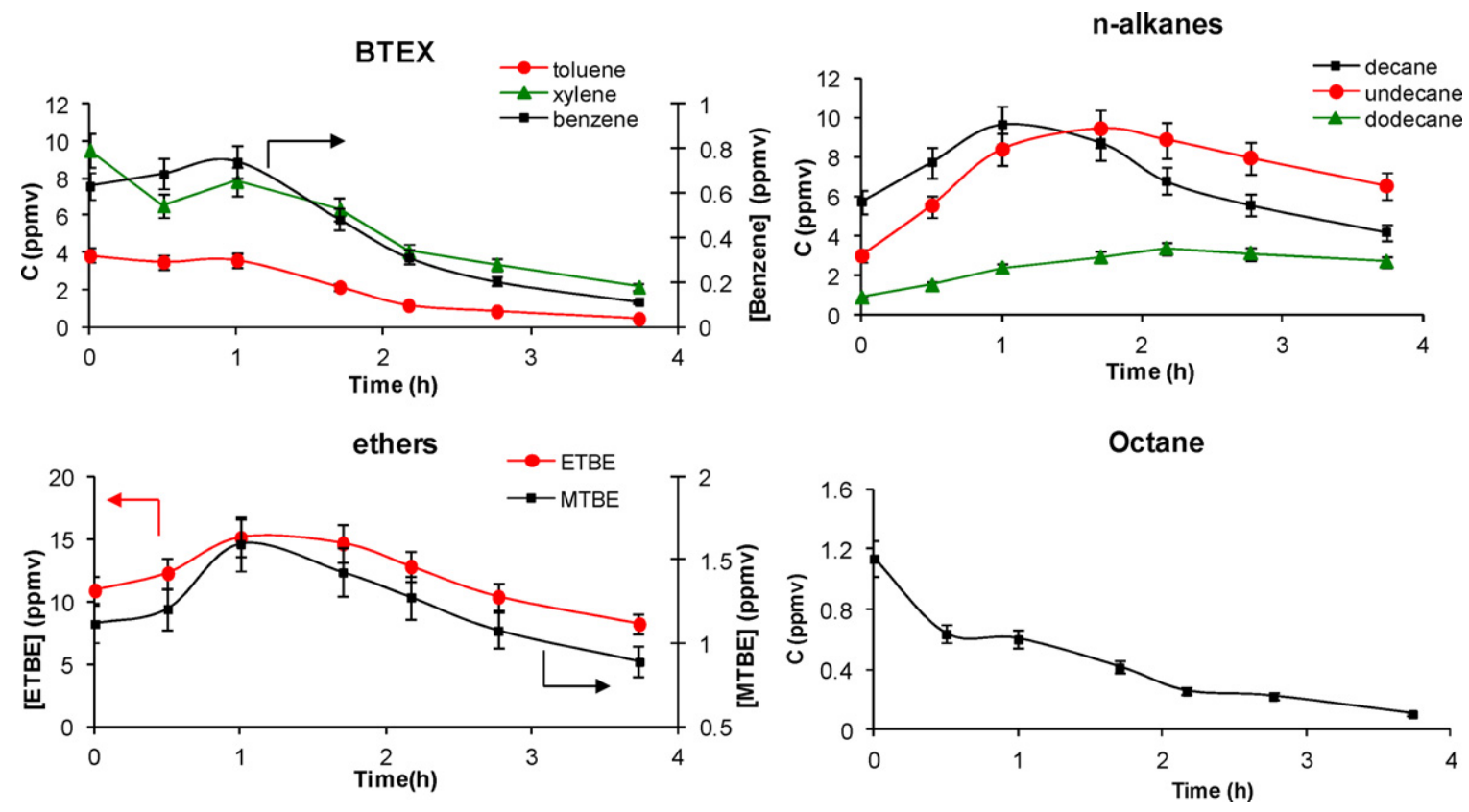

Fig. 7. Gaseous phase monitoring of the n-alkane, BTEX and ether concentrations.

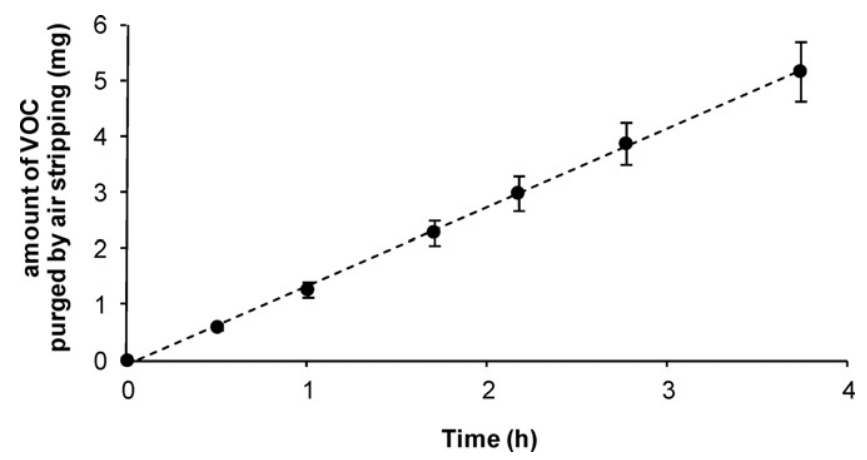

Fig. 8. Cumulative amount of VOCs purged from the aqueous phase into the gaseous one during the ASP treatement.

cannot access easier to the photocatalyst compared with how he can do it during a classical photocatalytic process. On the contrary, by purging decane and even the lightest molecules, undecane can improve its photocatalytic oxidation efficiency. Thus, by purging into the gaseous phase the most volatile dissolved compounds (i.e. those that are not concerned by MARPOL restrictions), we promote the degradation of the heaviest ones by photocatalysis and reduce the hydrocarbon oil index as defined in ISO 9377-2:2000 [30].

\subsubsection{Identification of the most resistant compounds}

Identification of the most resistant compounds to any treatment is pertinent for the study of their individual behaviour. Indeed, kinetic studies of these compounds may be of great importance with a view to dimensioning a pilot reactor for the real sample.
Fig. 9 shows the organic fraction (chromatogram extraction from $m / z=77$ and 91) that is still present after $24 \mathrm{~h}$ of treatment. It appears that all the aromatic compounds are eliminated at the end of the experiment (Fig. 9a). On the contrary, Fig. 9b indicates that, unless each individual concentration of n-alkanes (clearly identified with the black arrows) decreases, these compounds seem to be very persistent because their global contribution to the $[\mathrm{HC}]$ is quasi-constant with a slight increase (38\% vs. $34 \%$ initially). A solvent extraction of the photocatalytic paper indicates that this contribution even reaches $50 \%$ at the end of the treatment. Thus, it confirms that it is very difficult to eliminate these hydrophobic nalkanes from the bilgewater sample, and this is mainly due to their significant accumulation onto the photocatalytic paper because of their higher affinity with it in comparison with water. Moreover, it is confirmed in the literature that, although this may be possible, treatment of alkanes by photocatalysis is not easy, especially in the case of the linear ones [21].

In Fig. 10, we have compared the times required to remove $60 \%$ of the initial concentration of the different n-alkanes that are present in bilgewater (those with retention time $>\mathrm{RT}_{\text {decane }}$ ). The green line indicates the time required to reduce $[\mathrm{HC}]$ from 44 to $15 \mathrm{ppmv}$, i.e. the time required to remove up to $c a$. $60 \%$ of the initial [HC] $\left(t_{\mathrm{MARPOL}}\right)$. Surprisingly, it appears that $t_{60 \%}$ increases with $\mathrm{N}_{\mathrm{C}}$, the number of carbon atoms in the hydrocarbon chain. Nevertheless, we cannot compare our results with those published in the NIST database [34] nor with those reported by some authors $[35,36]$ which show exactly the opposite. With our experiments, we cannot access the intrinsic kinetics of degradation because of uncontrolled and limiting diffusion phenomena. Likewise, the concentration of each compound is not the same as can be seen in Fig. 2. As a consequence, the $t_{60 \%}$ expressed here represents

Table 3

Decane and undecane degradation in the case of the photocatalytic treatment and the ASP one: impact of photocatalysis on the total removal.

\begin{tabular}{|c|c|c|c|c|}
\hline \multirow[t]{2}{*}{$t=4 \mathrm{~h}$} & \multirow{2}{*}{$\begin{array}{l}\text { Photocatalysis } \\
\text { Removed by } \\
\text { photocatalysis (\%) }\end{array}$} & \multicolumn{3}{|c|}{ Air-stripping photocatalytic (ASP) treatment } \\
\hline & & $\begin{array}{l}\text { Purged by } \\
\text { air-stripping (\%) }\end{array}$ & $\begin{array}{l}\text { Removed by } \\
\text { photocatalysis (\%) }\end{array}$ & Total removal (\%) \\
\hline Decane & 23 & 60 & 24 & 84 \\
\hline Undecane & 16 & 38 & 43 & 80 \\
\hline
\end{tabular}



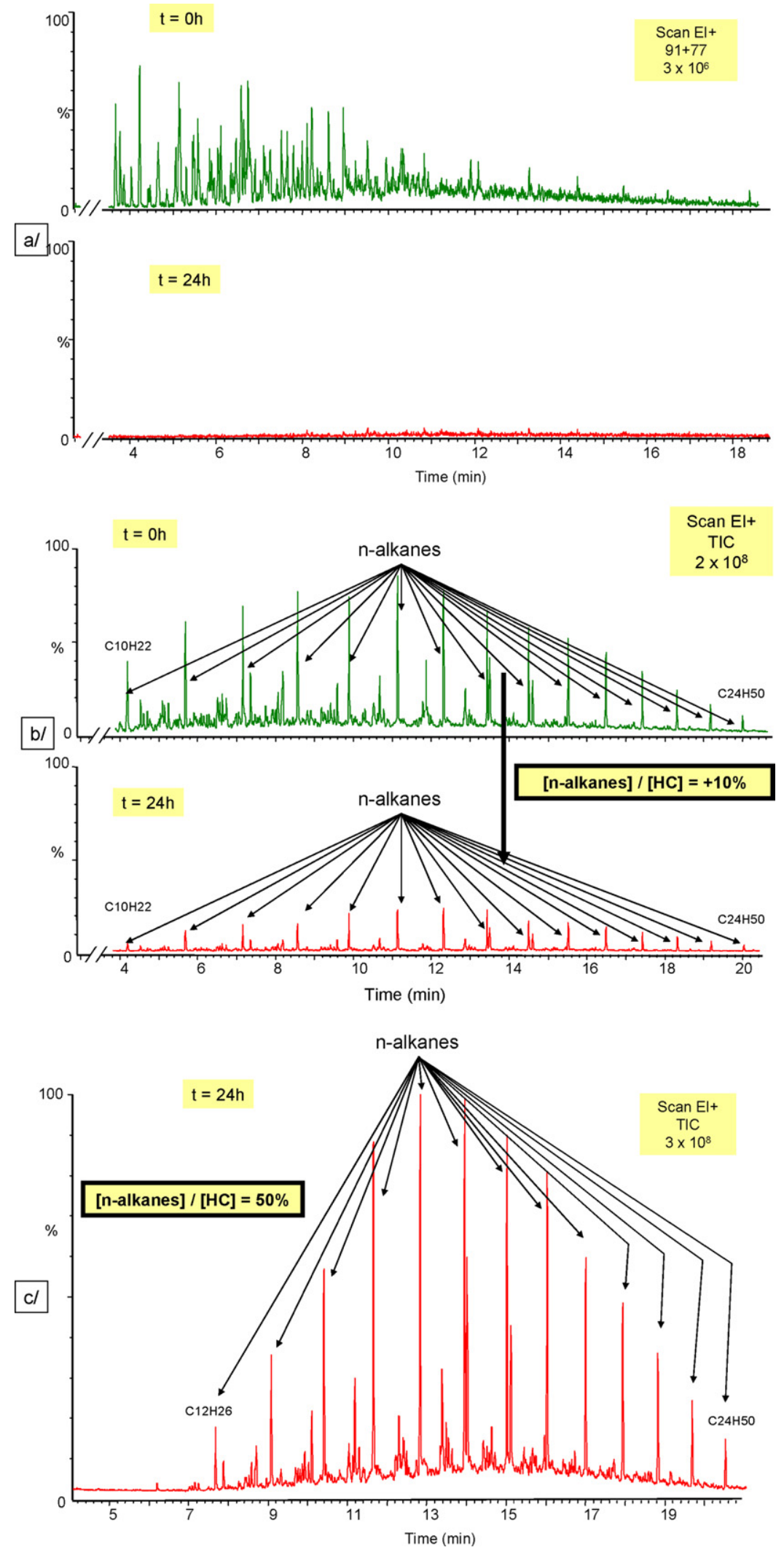

Fig. 9. Identification of the refractory compounds during the ASP treatment aromatics $(\mathrm{a} / \mathrm{)}$ and $\mathrm{n}$-alkanes $(\mathrm{b} / \mathrm{)}$ behaviour in the aqueous phase; (c/) n-alkane behaviour onto the photocatalytic paper. 


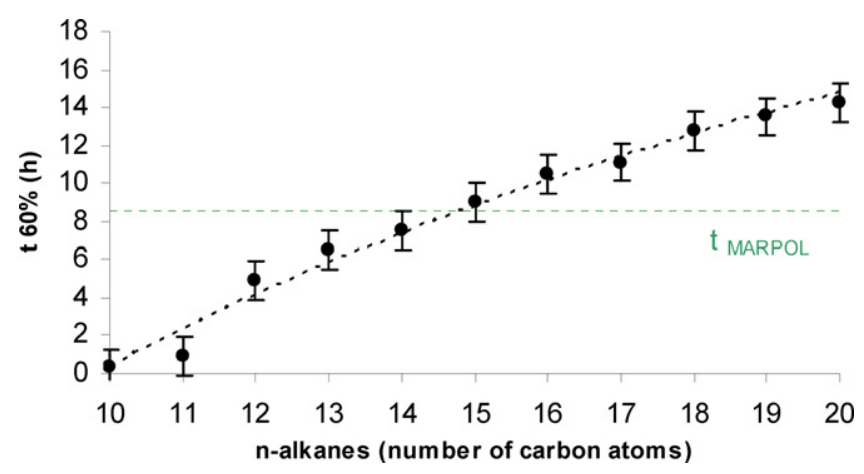

Fig. 10. Time required to remove $60 \%$ of the initial concentration of different nalkanes from decane to eicosane during ASP treatment of bilge water (green line: $t_{\mathrm{MARPOL}}=t_{[\mathrm{HC}]<15 \mathrm{ppm}}=t_{[\mathrm{HC}]_{60 \%}}$ ). (For interpretation of the references to color in this figure legend, the reader is referred to the web version of the article.)

an experimental observation that simultaneously describes all the kinetic steps (liquid-solid and liquid-gas diffusions, adsorption competitions, reactivity, etc.). Finally, we note that when $N_{C}$ is higher than 15 (pentadecane), $t_{60 \%}$ is even greater than $t_{\mathrm{MARPOL}}$. Thereby, it can be concluded that in order to treat bilgewater samples, the study of the n-alkanes with $\mathrm{N}_{\mathrm{C}}>15$ is very relevant since the rate of their removal slows down that of the overall process $([\mathrm{HC}])$.

\section{Conclusion}

The combination of air-stripping and photocatalysis has been studied for hydrocarbon removal from bilgewater. The aim of this treatment was to comply with MARPOL restrictions because the currently-proposed solutions are either insufficient or unsatisfactory (decantation, chemical treatment, etc.).

Results have shown that a good supply of air enhances the efficiency of the photocatalytic process and that a synergetic effect can be measured. By using this kind of combined process, we produced an effluent with $[\mathrm{HC}]<15 \mathrm{ppmv}$ (MARPOL) in only $8.5 \mathrm{~h}$ compared to the $17 \mathrm{~h}$ needed for a classical photocatalytic treatment. Indeed, in order to oxidize the organic materials, photocatalysis needs a minimum amount of dissolved oxygen and the supply of air in our case seems to be sufficient to overcome the lack of $\left[\mathrm{O}_{2}\right]$ in bilgewater samples. In addition, by purging the lightest hydrocarbons in the gaseous phase, aeration favours the diffusion toward the photocatalyst of the heaviest ones (and their degradation) which results in an increase of the $[\mathrm{HC}]$ removal efficiency.

In this study, we have also identified the compounds that are the most resistant to the process. When $\mathrm{N}_{C}>15$, n-alkanes are slowly removed and we have highlighted that they decrease the global rate of the treatment since they increase the time required to achieve the 15 ppmv limit defined by MARPOL. Thus, these compounds are of particular interest for the purpose of dimensioning a pilot plant reactor.

Finally, the analysis of the gaseous phase indicates that a lot of VOCs are purged from the aqueous phase. Even if most of them are not concerned by MARPOL (70\%), this kind of emission may be an environmental problem since a confined atmosphere like that of the bilge of ships would rapidly be polluted. With this in view, a double phase photoreactor in which both aqueous and gaseous phases can be treated is currently under study.

\section{Acknowledgment}

This work was supported by a Direction Générale de l'Armement (DGA) research grant. Especially, authors would like to thank M. Colombier and A. Giroud for their scientific contribution.
Authors would also like to thank Prakash Swamy who was a great help to us.

\section{References}

[1] International Maritime Organization (IMO), Resolution MEPC.117(52) Amendments to the annex of the protocol of 1978 relating to the international convention for the prevention of pollution from ships, 1973, 2000

[2] International Maritime Organization (IMO), MEPC.1/Circ.642 - 2008 revised guidelines for systems for handling oily wastes in machinery spaces of ships incorporating guidance notes for an integrated bilge water treatment system, 2008.

[3] M. Tomaszewska, A. Orecki, K. Karakulski, Treatment of bilge water using a combination of ultrafiltration and reverse osmosis, Desalination 185 (2005) 203-212.

[4] M. Cheryan, N. Rajagopalan, Membrane processing of oily streams - wastewater treatment and waste reduction, J. Memb. Sci. 151 (1998) 13-28.

[5] R. Ghidossi, D. Veyret, J.L. Scotto, T. Jalabert, P. Moulin, Ferry oily wastewater treatment, Sep. Purif. Technol. 64 (2009) 296-303.

[6] J.A. Caplan, C. Newton, D. Kelemen, Technical report: novel oil/water separator for treatment of oily bilgewater, Mar. Technol. SNAME News 37 (2000) 111-115.

[7] M.L. Nievas, M.G. Commendatore, N.L. Olivera, J.L. Esteves, V. Bucala, Biodegradation of bilge waste from Patagonia with an indigenous microbial community, Bioresour. Technol. 97 (2006) 2280-2290.

[8] C. Sivaraman, A. Ganguly, S. Mutnuri, Biodegradation of hydrocarbons in the presence of cyclodextrins, World J. Microbiol. Biotechnol. 26 (2010) 227-232.

[9] J.L. Bernal, J.R.P. Miguelez, E.N. Sanz, E.M. de la Ossa, Wet air oxidation of oily wastes generated aboard ships: kinetic modeling, J. Hazard. Mater. 67 (1999) 61-73.

[10] M. Asselin, P. Drogui, S.K. Brar, H. Benmoussa, J.F. Blais, Organics removal in oily bilgewater by electrocoagulation process, J. Hazard. Mater. 151 (2008) 446-455.

[11] B.K. Korbahti, K. Artut, Electrochemical oil/water demulsification and purification of bilge water using $\mathrm{Pt} / \mathrm{Ir}$ electrodes, Desalination 258 (2010) 219-228.

[12] K. Karakulski, W.A. Morawski, J. Grzechulska, Purification of bilge water by hybrid ultrafiltration and photocatalytic processes, Sep. Purif. Technol. 14 (1998) 163-173.

[13] A. Lair, Etude de la Dégradation des Hydrocarbures par Photocatalyse: Application au Traitement des Eaux de Cales, PhD, Claude Bernard University, Lyon 1, Lyon, 2007.

[14] J.-M. Herrmann, Heterogeneous photocatalysis: fundamentals and applications to the removal of various types of aqueous pollutants, Catal. Today 53 (1999) 115-129.

[15] S. Malato, P. Fernández-Ibáñez, M.I. Maldonado, J. Blanco, W. Gernjak, Decontamination and disinfection of water by solar photocatalysis: recent overview and trends, Catal. Today 147 (2009) 1-59.

[16] M.N. Chong, B. Jin, C.W.K. Chow, Chris Saint, Recent developments in photocatalytic water treatment technology: a review, Water Res. 44 (2010) 2997-3027.

[17] B. Ohtani, Photocatalysis, A to Z-what we know and what we do not know in a scientific sense, J. Photochem. Photobiol. C: Photochem. Rev. 11 (2010) 157-178.

[18] M. Sturini, F. Soana, A. Albini, Reaction paths in the titanium dioxide photocatalysed degradation of dodecane and some of its derivatives, Tetrahedron 58 (2002) 2943-2950.

[19] E. Vulliet, C. Emmelin, J.-M. Chovelon, C. Guillard, J.-M. Herrmann, Photocatalytic degradation of sulfonylurea herbicides in aqueous $\mathrm{TiO}_{2}$, Appl. Catal. B: Environ. 38 (2002) 127-137.

[20] C. Guillard, P. Theron, P. Pichat, C. Petrier, Evaluation of 1-octanol degradation by photocatalysis and ultrasound using SPME, Water Res. 36 (2002) 4263-4272.

[21] M. D’Auria, L. Emanuele, R. Racioppi, V. Velluzzi, Photochemical degradation of crude oil: comparison between direct irradiation, photocatalysis, and photocatalysis on zeolite, J. Hazard. Mater. 164 (2009) 32-38.

[22] R.L. Ziolli, W.F. Jardim, Photocatalytic decomposition of seawater-soluble crude-oil fractions using high surface area colloid nanoparticles of $\mathrm{TiO}_{2}$, J. Photochem. Photobiol. A: Chem. 147 (2002) 205-212.

[23] C. Minero, V. Maurino, E. Pelizzetti, Photocatalytic transformations of hydrocarbons at the sea water air interface under solar radiation, Mar. Chem. 58 (1997) 361-372.

[24] M. Subramanian, A. Kannan, Effect of dissolved oxygen concentration and light intensity on photocatalytic degradation of phenol, Korean J. Chem. Eng. 25 (2008) 1300-1308.

[25] J. Staudinger, P.V. Roberts, A critical compilation of Henry's law constant temperature dependence relations for organic compounds in dilute aqueous solutions, Chemosphere 44 (2001) 561-576.

[26] M.C. Kavanaugh, R.R. Trussell, Design of aeration towers to strip volatile contaminants from drinking-water, J. Am. Water Works Assoc. 72 (1980) 684-692.

[27] T.C.G. Kibbey, Kurt D. Pennell, Kim F. Hayes, Application of sieve-tray air strippers to the treatment of surfactant-containing wastewaters, AIChE J. 47 (2001) 1461-1470. 
[28] H.F. Cheng, Y.N. Hu, J. Luo, D.A. Sabatini, Multipass membrane air-stripping (MAS) for removing volatile organic compounds (VOCs) from surfactant micellar solutions, J. Hazard. Mater. 170 (2009) 1070-1078.

[29] International Maritime Organization (IMO), Resolution MEPC.107(49)- revised guidelines and specifications for pollution prevention equipment for machinery space bilges of ships, 2003.

[30] International Organization of Standardization (ISO), ISO 9377-2:2000. Water quality - determination of hydrocarbon oil index - Part 2: Method using solvent extraction and gas chromatography, 2000.

[31] K.K. Turekian, Oceans, Prentice-Hall ed., 1968

[32] E.E. Moyer, P.T. Kostecki, MTBE Remediation Handbook, Springer ed., 2003.
[33] L.L.P. Lim, R.J. Lynch, Feasibility study of a photocatalytic reactor for in situ groundwater remediation of organic compounds, J. Hazard. Mater. 194 (2011) $100-108$.

[34] NIST, NDRL/NIST Solution Kinetics Database, 2002. http://kinetics. nist.gov/solution/.

[35] N. Djeghri, M. Formenti, F. Juillet, S.J. Teichner, Photointeraction on surface of titanium-dioxide between oxygen and alkanes, Faraday Dis. 58 (1974) 185-193.

[36] A.K. Boulamanti, C.J. Philippopoulos, Photocatalytic degradation of C5-C7 alkanes in the gas-phase, Atmos. Environ. 43 (2009) 3168-3174. 